\title{
Application of Machine Learning for Dragline Failure Prediction
}

\author{
Amir Taghizadeh ${ }^{1}$, Nuray Demirel ${ }^{{ }^{*}}$ \\ ${ }^{1}$ Mining Engineering Department, Middle East Technical University, Üniversiteler Mah., Dumlupınar \\ Bulvarı, No: 1, 06800 Çankaya, Ankara, Turkey
}

\begin{abstract}
Overburden stripping in open cast coal mines is extensively carried out by walking draglines. Draglines' unavailability and unexpected failures result in delayed productions and increased maintenance and operating costs. Therefore, achieving high availability of draglines plays a crucial role for increasing economic feasibility of mining projects. Applications of methodologies which can forecast the failure type of dragline based on the available failure data not only help to reduce the maintenance and operating costs but also increase the availability and the production rate. In this study, Machine Learning approaches have been applied for data which has been gathered from an operating coal mine in Turkey. The study methodology consists of three algorithms as: i) implementation of K-Nearest Neighbors, ii) implementation of MultiLayer Perceptron, and iii) implementation of Radial Basis Function. The algorithms have been utilized for predicting the draglines' failure types. In this sense, the input data, which are mean time-to-failure, and the output data, failure types, have been fed to the algorithms. The regression analysis of methodologies have been compared and showed the K- Nearest Neighbors has a higher rate of regression which is around 70 percent. Thus, the K-Nearest Neighbor algorithm can be applied in order to preventive components replacement which causes to minimized preventive and corrective cost parameters. The accurate prediction of failure type, indeed, causes to optimized number of inspections. The novelty of this study is application of machine learning approaches in draglines' reliability subject for first time.
\end{abstract}

\section{Introduction}

Draglines work in a harsh working environment and prone to various failures as they operate. Lack of proper maintenance and investigation may increase the rate of failures and unavailability (Ebeling, 2010). A failure takes a place in a subsystem of a dragline halts the whole machine out of operation. Therefore, proper failure analysis and predicting the failures in advance may help in optimization of dragline preventive maintenance and eventually increase the availability and reliability of the system.

\footnotetext{
* Corresponding author: ndemirel@metu.edu.tr
} 
The availability definition is equal with performability; moreover, quality, reliability, and maintainability should be considered together to assess the preformability. In order to achieve high performability of the system, appropriate and effective preventive and corrective actions should be taken on time. Corrective maintenance has to be carried out to recover system to its functional state. On the contrary side, preventive maintenance is utilized for forecasting failure(s) and its types as well. In this case, a predictor, which has been modeled based on the preventive step, benefits to repair, replace or make investigation for a component in a system. Since the preventive action can diminish the maintenance cost, which has 40 to 50 percent of the equipment operating cost in a mine (Forsman et al, 1992), appropriate prediction of failure types led to lower not only equipment operating cost but also total operation cost of a mine (i.e. maintenance cost is around to 20 to 35 percent of overall operating cost of a mine (Unger et al., 1994).

In the mining industry like others, the systems breakdown has its effects particularly on production rate, time losses, and safety issue. Based on literature survey, the draglines, as high-capacity complex machines, are bottlenecks of mines, so availability of the mentioned excavator are vital. In this sense, reliability measurement of a dragline should be taken into consideration. Hence, various types of methods have been utilized in order to assess the reliability of a system such as: Effects and Criticality Analysis (FMECA), Fault Tree Analysis (FTA), Failure Modes, Markov Analysis (MA), Reliability Block Diagram (RBD). Since all the named methods are based on conventional linear algebra or statistical algorithms, the utilization of them in a domain which has high volume of data is impractical.

In order to handle the mentioned objective, machine learning approaches which use for data with high volume as well as high frequency are utilized. In this study, machine learning approaches (i.e. Multi-Layer Perceptron, Radial Basis Function, and K-Nearest Neighbor) have been implemented on a dataset which was shaped by gathered data from a dragline currently operating in a coal mine, Turkey. The utilized methods which are categorized as classification algorithms have one feature and 1,303 observations. There are six different failure types were identified in the dataset. Table 1 tabulates the causes of dragline failures and failure numbers associated with each type. The input data are Time to Failure (TTF) and the output data are called types of breakdowns. Table 2 presents sample datasets used as input for the algorithm.

Table 1. Causes and number of dragline failures

\begin{tabular}{|c|c|c|}
\hline ID of Failure Type & Failure Type & Failure Number \\
\hline 1 & mechanical failures & 945 \\
\hline 2 & electrical failures & 276 \\
\hline 3 & energy related failures & 53 \\
\hline 4 & major revision & 5 \\
\hline 5 & maintenance & 14 \\
\hline 6 & power cut & 7 \\
\hline
\end{tabular}

Table 2. Sample dataset

\begin{tabular}{|c|c|}
\hline Mean Time to Failure (Hour) & ID of Failure Type \\
\hline 1.6597 & 1 \\
\hline 3.5625 & 1 \\
\hline 14.4167 & 2 \\
\hline 5.5625 & 6 \\
\hline 0.5938 & 1 \\
\hline
\end{tabular}




\section{Application of Machine Learning Algorithms for Dragline Failure Prediction}

The assessment of draglines' reliability aims to achieve high availability which is a crucial task in the mining industry. Based on idea mentions practical engineering system, which is not like a closed-form relation, encompasses various types of components, so the elaborated calculation is required. In this sense, Machine Learning (ML) approaches, which can model the complex system, can be utilized. Modeling the whole system in respect to assess the reliability in the case of quantitative data by means of ML is not only effective but also operational and logical action in case of big data management.

ML approaches have been implemented in the recent studies (Hurtado et al. (2001), Xu et al. (2002), Hurtado (2004), Zhiwei (2008), Gomes et al. (2004), Schueremans et al. (2005), Roco et al. (2002), Chua (2002)). Since the abilities of parallel processing with respect to learning, generalizing to classify, and organizing data is the main reason for using Artificial Neural Network (ANN) in this study. ANN models have been categorized to two groups: supervised training and unsupervised training. The structure of ANN, also, can divided to two parts feedback and feedforward architecture (i.e. in some cases combination of two architectures might be used). In this study, Multi-Layer Perceptron (MLP) and Radial Basis Function (RBF) algorithms as methods of ANN, which are supervised training methods, have been utilized (i.e. ANN is an approach of ML). MLP and $\mathrm{RBF}$ have been implemented on data which were acquisitioned from an operating dragline in a coal mine in 23 years. The arrays of pre-processing methods have been carried out on the dataset, such as: i) data cleaning, ii) data integration, and iii) data transformation. In order to tackle the missing values, which is located in data cleaning phase, data have been eliminated. The idea of MLP is in simplest format is close meaning of logistic regression, which contains one hidden layer and only a single neuron (Figure 1).

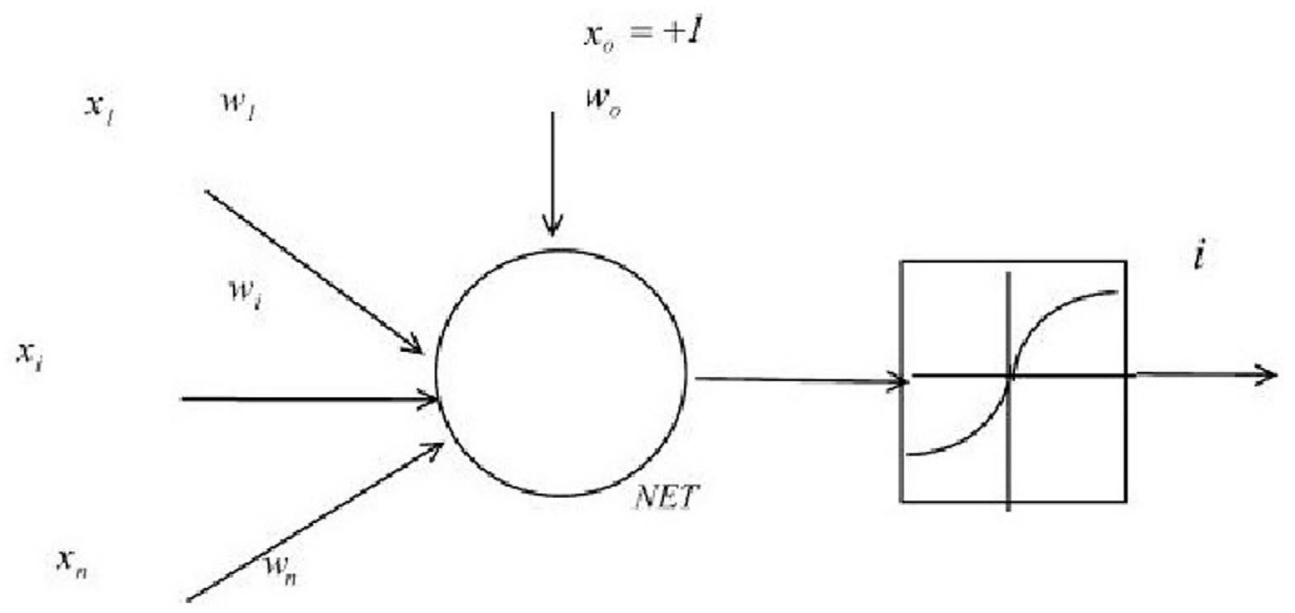

Fig. 1. Single neuron MLP

A single neuron unit was fed by input vector(s); then some calculation which is called transformation is carried out on inputs to reach output vector(s). Transformation was carried out in two stages, i) sum up of all inputs $\left(\mathrm{x}_{\mathrm{i}}\right)$ and their corresponding weights $\left(\mathrm{w}_{\mathrm{i}}\right)$, so the scalar is going to be calculated and ii) the output(s) based on the scalar and an activation function (i.e. an activation function can be linear or non-linear) is generated. A single processing unit makes connections with other processing units, so a Neural Network is shaped. In a network, it is necessary for weights to be adjusted. In order for weights adjusting, Gradient Descent method as an optimizer of weights was applied in this study. 
An input layer and an output layer get their data from a network. In shaping a Neural Network, it is necessary to determine the number of hidden layer as well as the number of hidden units. Since a model is more complex, more hidden layer is required; moreover, the number of hidden units is determined by trial and error in practice (Fig 2).

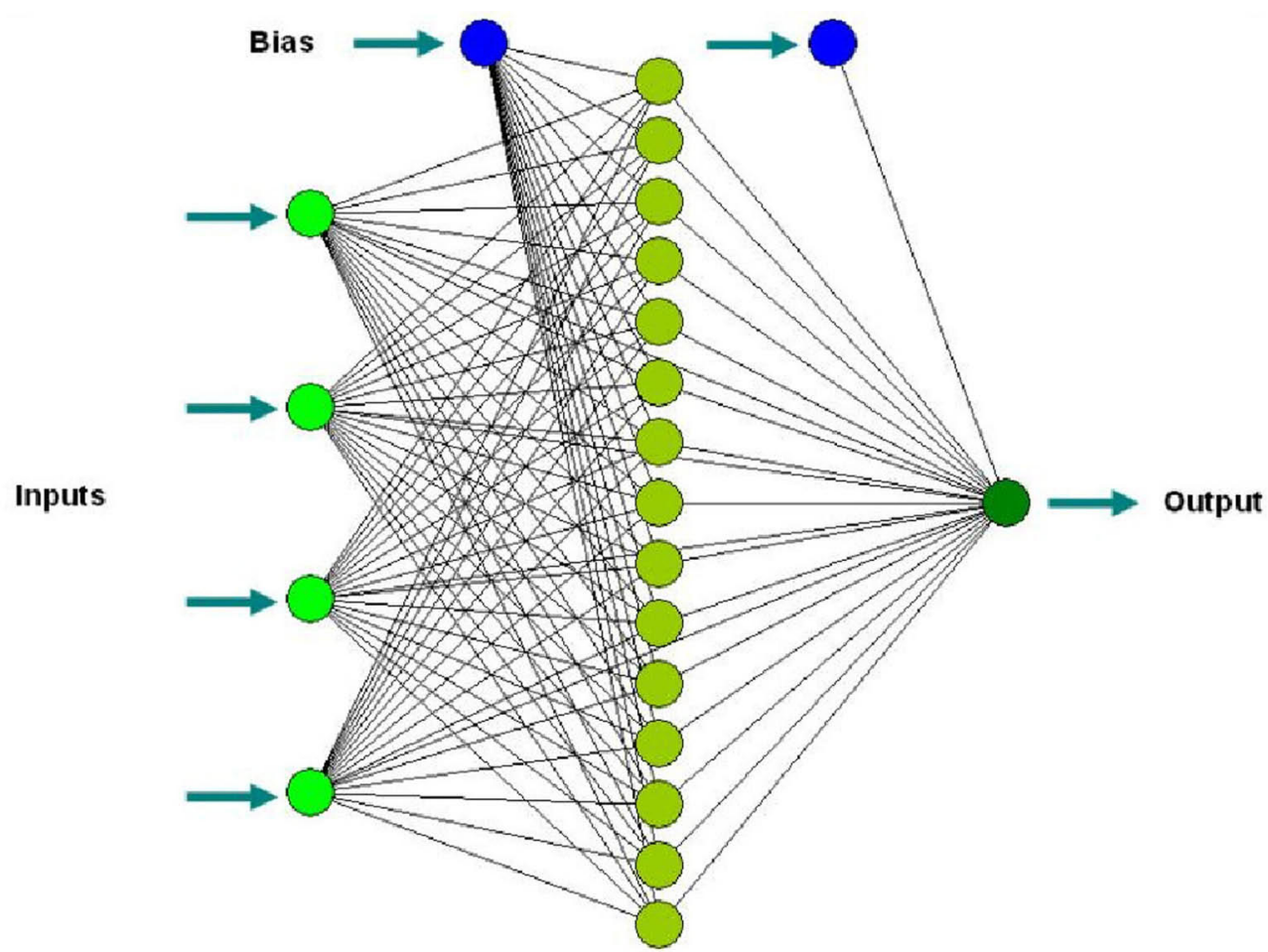

Fig. 2. Schematic view of multi-layer perceptron network

In this study, MLP with four hidden layers and five processing neuron in each layer has been utilized. The regression analysis of MLP is 37 percent; moreover, the Root Mean Square Error (RMSE) for MLP is 0.79. In the case of high RMSE and low regression analysis of MLP, RBF has been implemented on dataset. In RBF, input vector(s) feed to a Neural Network and instead of weight which taken in Perceptron neurons, the Euclidean Norm is calculated (i.e. the Euclidean Norm is distance between input vector(s) and center vector). The multiplication of Euclidean result and a threshold (b) is feed to non-linear function (i.e. the radial basis function (G)) (Fig 3). The calculation is shown in equation below:

$$
a=(\|x-t\|) \bullet b
$$




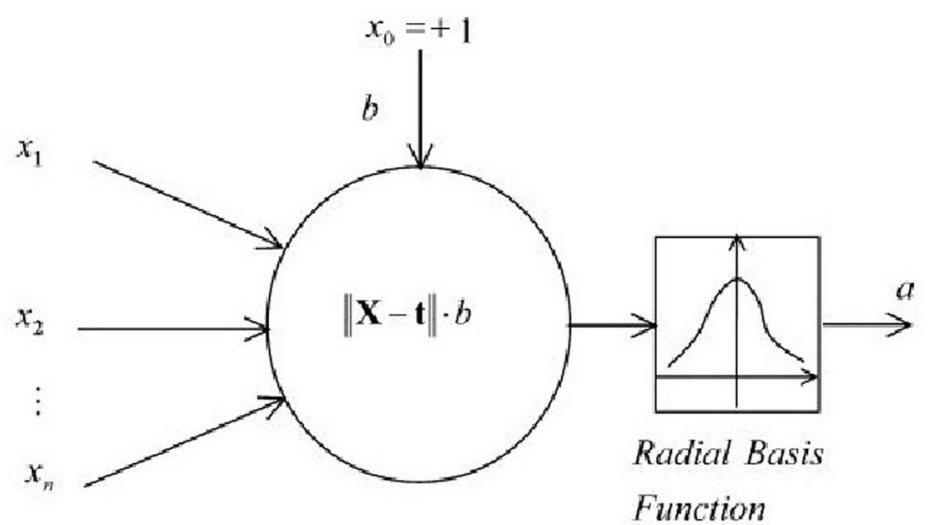

Fig. 3. Single radial basis function

When number of single radial basis function connected each other, the Neural Network of radial basis function shaped (Fig. 4).

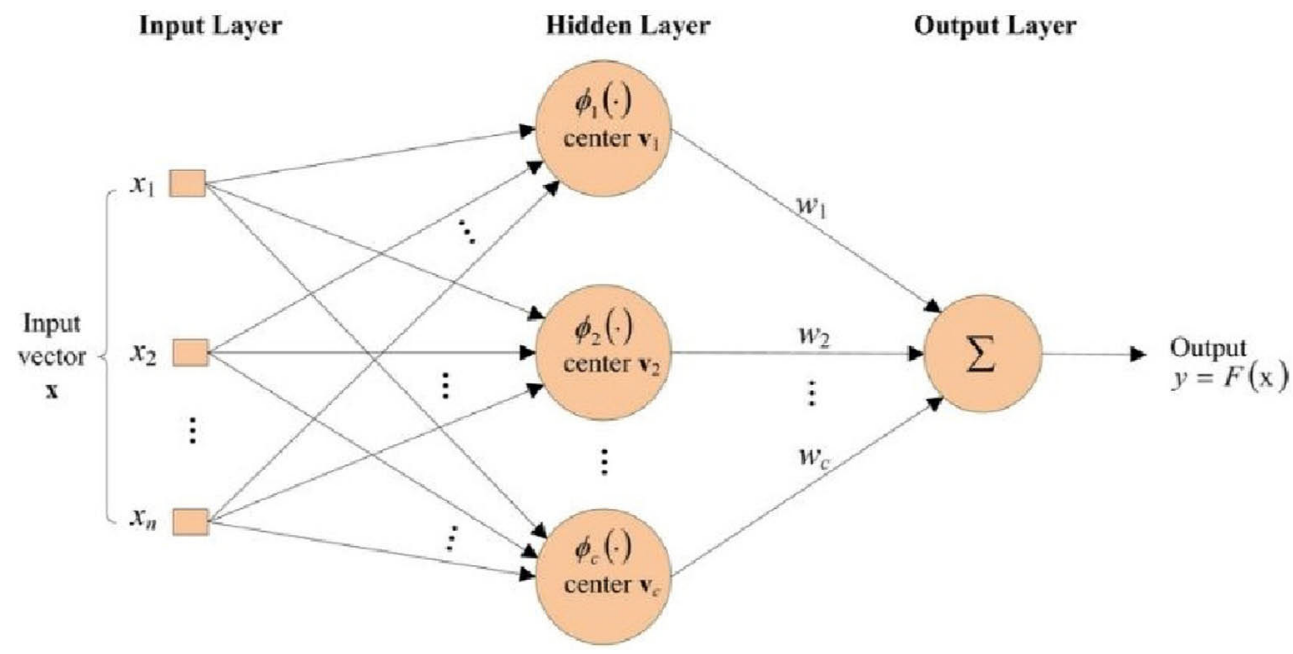

Fig. 4. Schematic view of radial base function network.

The regression analysis for RBF is 18 percent and RMSE is 0.74 . Since MLP could not achieve the desired result, nor did RBF, K- Nearest neighbor algorithm has been utilized in order to achieve a result with higher regression analysis.

K-Nearest Neighbor (KNN) is non-parametric algorithm which is called lazy algorithm. Since it does not get any assumptions on the data distribution, it is categorized as nonparametric algorithm. In this sense, the application of KNN in the practical data, which does not have any typical assumptions, can be profitable. While KNN does not utilize any training data in order to do generalization, it is called Lazy algorithm. In the KNN algorithm, decision is made based on whole of training dataset. Therefore, KNN requires more time as well as more memory in order to make a decision. Since, data is in a feature space, the notion of distance exist, which can be Euclidean distance or any common used distance measuring algorithm. In the KNN algorithm, every data in feature space has label, in the simplest trend number of classes are to two. However, KNN works with uninformed number of classes. Based on the distance matrix the number of neighbor $(\mathrm{K})$ is calculated. In this study, KNN has been applied for the dataset, and therefore, it yields regression 
analysis 72 percent. Table 3 presents the utilized machine learning algorithms and respective regression analysis results.

Table 3. Utilized ML algorithm and results

\begin{tabular}{|c|c|}
\hline Algorithm & Regression Analysis (\%) \\
\hline Multi-Layer Perceptron & 37 \\
\hline Radial Basis Function & 18 \\
\hline K-Nearest Neighbour & 72 \\
\hline
\end{tabular}

\section{Conclusions}

Draglines, as a single operating machine in the coal mines, play a crucial role. In this sense, reliability of draglines should be taken into consideration, since it breakdown of the mentioned mining machinery causes losses in time and profit as well as higher direct and indirect costs. In this study, three types of machine learning approaches have been utilized in order to predict the failure type of a dragline and to make a predictive model of breakdown, so higher rate of regression analysis can fulfill the better prediction with respect to prognostic process in condition-based maintenance. Mean time to failure and failure type as the input vector and the output vector has been used, respectively. Three algorithms have been implemented on the dataset. As a result, K-nearest neighbor algorithm has a better result in contrast to other utilized algorithm. This study showed that machine learning approaches can effectively be utilized to predict the failure type of a dragline. As a conclusion, preventive maintenance policies should adopt machine learning algorithms to achieve optimized maintenance programs which yields lower maintenance costs, fewer machine failures, less repair downtime, reduce small parts inventory, longer life of dragline, increase of its production and improved operator safety.

\section{References}

1. K. S. Chua, Pattern Recognition Letters, 24, 75 (2003)

2. C.E. Ebeling, An introduction to reliability and maintainability engineering. (Waveland Press Inc, 2010)

3. B. Forsmann and U. Kumar, Journal of Mines, Metals and Fuels, 40, 267 (1992)

4. H. M. Gomes and A. M. Awruch, Structural Safety. 26, 49 (2004)

5. J. E. Hurtado, Structural Safety. 26. 271 (2004)

6. J. E. Hurtado and D. A. Computer Methods in mechanics and Engineering. 191, 113 (2001)

7. C. M. Rocco and J. A. Moreno, Reliability Engineering and System Safety. 76, 237 (2002)

8. L. Schueremans and D. V. Gemert, Structural Safety. 27, 246 (2005)

9. R. L. Unger and K. Conway, Impact of Maintainability Design on Injury Rates and Maintenance Costs for Underground Mining Equipment. In R. H. Peters, Special Publication Report, 140 (1994)

10. G. Zhiwei and B. Guangchen, Chinese Journal of Aeronautics. 22, 160 (2009) 